\title{
PRESENTATIONS OF FAITHFUL D.G. NEAR-RINGS
}

\author{
by J. D. P. MELDRUM \\ (Received 15th September 1978)
}

\section{Introduction}

Many basic definitions and results in the theory of near-rings can be found in $G$. Pilz (4). We follow these for the most part, except that we use left near-rings rather than right near-rings. We follow exactly an earlier paper, Meldrum (2), where there are detailed definitions and many results relating to faithful d.g. near-rings. Let $R$ be a d.g. near-ring, distributively generated by the semigroup $S$, which need not be the semigroup of all distributive elements. Denote such a d.g. near-ring by $(R, S)$. Then $(R,+)=G p<S ; \mathscr{R}>$ where $\mathscr{R}$ is a set of defining relations in $S$. Let $(T, U)$ be a d.g. near-ring. Then a d.g. homomorphism $\theta$ from $(R, S)$ to $(T, U)$ is a near-ring homomorphism from $R$ to $T$ which satisfies $S \theta \subseteq U$. If $(G,+)$ is a group, let $T_{0}(G)$ be the near-ring of all maps from $G$ to itself with pointwise addition and map composition. Let End $G$ be the semigroup of all endomorphisms of $G$. Then $(E(G)$, End $G)$ is a d.g. near-ring. A d.g. near-ring $(R, S)$ is faithful if there exists a d.g. monomorphism $\theta:(R, S) \rightarrow(E(G)$, End $G)$ for some group $G$.

We now consider free $(R, S)$ groups on a set $X$. For any d.g. near-ring, the free $(R, S)$ group on a set $X$ exists and is denoted $\operatorname{Fr}(X, R, S)$ (Section 2 of Meldrum (2)). If $X=\{x\}$ is a one element set, we write $\operatorname{Fr}(x, R, S)$ for $\operatorname{Fr}(X, R, S)$. A group $G$ is an $(R, S)$ group if there exists a representation $\theta:(R, S) \rightarrow(E(G)$, End $G)$, a d.g. homomorphism defining an action of $(R, S)$ on $G$. In this context free has its usual universal meaning. The lower faithful d.g. near-ring for $(R, S)$ is a faithful d.g. near-ring $(\underline{R}, \underline{S})$ such that $\underline{\theta}:(R, S) \rightarrow(\underline{R}, \underline{S})$ is a d.g. epimorphism and $S \underline{\theta}=\underline{S}$. Further if $\theta$ is any d.g. homomorphism from $(R, S)$ to a faithful d.g. near-ring $(T, U)$, then $\theta=\underline{\theta} \phi$ for a uniquely defined d.g. homomorphism $\phi$. Lower faithful d.g. near-rings always exist, as was shown in Meldrum (2), Theorem 4.3 and Mahmood (1), Theorem 2.1. We quote this result as follows.

Theorem 1.1. Let $(R, S)$ be a d.g. near-ring. Then its lower faithful near-ring is $(\underline{R}, \underline{S})=(R, S) / A$, where $A=\{r \in R ; G r=0\}$, and $G$ is the free $(R, S)$ group on one generator.

In this paper, we consider d.g. near-rings $(R, S)$. Then $(R,+)$ has a presentation of the form $G p<S ; \mathscr{R}>$, where $\mathscr{R}$ is a set of defining relations. We find conditions on the presentation which are equivalent to the statement that $(R, S)$ is a faithful d.g. near-ring. This is then used to find the lower faithful d.g. near-ring in some simple cases. 
Finally we quote another result, Lemma 4.4. of Meldrum (2), which we will need later.

Theorem 1.2. Let $(R, S)$ be a faithful d.g. near-ring, and let $G=\operatorname{Fr}(x, R, S)$. Then $G=\mathrm{Gp}\langle x\rangle *(R,+)$ is the free product of an infinite cyclic group with a group isomorphic to $(R,+)$.

We- remark that we could restrict ourselves to working within a variety $\mathscr{V}$ of additive groups as was done in Meldrum (2), and all the results will go over in such a context.

\section{Faithful presentations}

Definition 2.1. We call a presentation

$$
(R,+)=\mathrm{Gp}\langle S ; \mathscr{R}\rangle
$$

a faithful presentation if $(R, S)$ is a faithful d.g. near-ring.

Let us consider such a presentation of a d.g. near-ring $(R, S)$, which is not necessarily faithful. To simplify the statement of the next result, let $\mathbf{u}=\left(u_{1}, \ldots, u_{r}\right)$ be an $r$-tuple of elements of $S, \mathbf{u}^{\prime}=\left(u_{1}^{\prime}, \ldots, u_{t}^{\prime}\right)$ be a $t$-tuple of elements of $S$, and let $w(u), v\left(\mathbf{u}^{\prime}\right)$ be two words in $\left(u_{1}, \ldots, u_{r}\right)$ and $\left(u_{1}^{\prime}, \ldots, u_{t}^{\prime}\right)$ respectively. The following result is a key technical lemma.

Lemma 2.2. With the above notation,

$$
w(\mathbf{u}) \cdot v\left(\mathbf{u}^{\prime}\right)=v\left(w(\mathbf{u}) \cdot \mathbf{u}^{\prime}\right)
$$

where

$$
w(\mathbf{u}) \cdot \mathbf{u}^{\prime}=\left(w\left(\mathbf{u} u_{1}^{\prime}\right), \ldots, w\left(\mathbf{u} u_{\imath}^{\prime}\right)\right)
$$

and

$$
\mathbf{u} u_{j}^{\prime}=\left(u_{1} u_{j}^{\prime}, \ldots, u_{r} u_{j}^{\prime}\right)
$$

Proof. We use a double induction on the length $n$ of the word $w$, and the length $m$ of the word $v$. First assume that $n=1=m$. Then $w(\mathbf{u})=\epsilon_{1} u_{1}, v\left(\mathbf{u}^{\prime}\right)=\eta_{1} u_{1}^{\prime}$, where $\epsilon_{1}$ and $\eta_{1}$ are \pm 1 , and $w(\mathbf{u}) \cdot v\left(\mathbf{u}^{\prime}\right)=\epsilon_{1} u_{1} \cdot \eta_{1} u_{1}^{\prime}=\epsilon_{1} \eta_{1} u_{1} u_{1}^{\prime}=\eta_{1}\left(\epsilon_{1}\left(u_{1} u_{1}^{\prime}\right)\right)$. So the result is true for $n=1=m$. Now assume that the result is true for $n=p, m=1$, and let the length of $w(u)$ be $p+1$. Then $w(u)=w^{\prime}(u)+\epsilon u_{i}$, where $\epsilon= \pm 1$, and the length of $w^{\prime}(u)$ is $p$. Then

$$
\begin{aligned}
w(\mathbf{u}) \cdot \eta_{1} u_{1}^{\prime} & =\left(w^{\prime}(\mathbf{u})+\epsilon u_{i}\right) \eta_{1} u_{1}^{\prime} \\
& =\eta_{1}\left(\left(w^{\prime}(\mathbf{u})+\epsilon\left(u_{i}\right)\right) u_{1}^{\prime}\right) \\
& =\eta_{1}\left(\left(w^{\prime}(\mathbf{u}) u_{1}^{\prime}+\epsilon\left(u_{i} u_{1}^{\prime}\right)\right) \text { as } u_{1}^{\prime}\right. \text { is distributive } \\
& =\eta_{1}\left(w^{\prime}(\mathbf{u}) \cdot u_{1}^{\prime}+\epsilon\left(u_{i} u_{1}^{\prime}\right)\right) \text { by the induction hypothesis } \\
& =\eta_{1} w(\mathbf{u}) \cdot u_{1}^{\prime}=v\left(w(\mathrm{u}) \cdot u_{1}^{\prime}\right) .
\end{aligned}
$$

This proves the result for all $n \geqslant 1$ and $m=1$. 
Now assume that the result is true for $m=q$ and let the length of $v\left(\mathbf{u}^{\prime}\right)=q+1$. Then $v\left(\mathrm{u}^{\prime}\right)=v^{\prime}\left(\mathrm{u}^{\prime}\right)+\eta u_{j}^{\prime}$, where $\eta= \pm 1$ and the length of $v^{\prime}\left(\mathrm{u}^{\prime}\right)$ is $q$. Then

$$
\begin{aligned}
w(\mathbf{u}) \cdot v\left(\mathbf{u}^{\prime}\right) & =w(\mathbf{u}) \cdot\left(v^{\prime}\left(\mathbf{u}^{\prime}\right)+\eta u_{j}^{\prime}\right) \\
& =w(\mathbf{u}) \cdot v^{\prime}\left(\mathbf{u}^{\prime}\right)+w(\mathbf{u}) \cdot \eta u_{j}^{\prime} \text { by left distributivity } \\
& =v^{\prime}\left(w(\mathbf{u}) \cdot \mathbf{u}^{\prime}\right)+\eta w(\mathbf{u}) \cdot u_{j}^{\prime} \text { by the induction hypothesis } \\
& =v\left(w(\mathbf{u}) \cdot \mathbf{u}^{\prime}\right) .
\end{aligned}
$$

This finishes the double induction and proves the result.

We now make precise the criterion for faithfulness in a d.g. near-ring which we will use.

Theorem 2.3. The d.g. near-ring $(R, S)$ is faithful if and only if an identity 1 can be adjoined to $R$ in such a way that the elements of $S$ remain distributive in the larger near-ring, $(T, U)$ say, where $U=S \cup\{1\}$. Then $(T,+)=\mathrm{Gp}\langle 1\rangle * \mathrm{Gp}\langle S\rangle$, where * indicates free product.

The first part of this result is a well-known characterisation of faithfulness applied to this case. The second part follows from Theorem 1.2 or from Meldrum (2), Lemma 4.7. Now assume that $(R, S)$ has a presentation as in Definition 2.1 , and is faithful. Let the relations be

$$
\mathscr{R}=\left\langle\boldsymbol{r}_{\Lambda}\left(\mathbf{s}^{(\lambda)}\right) ; \lambda \in \Lambda\right\}
$$

By Theorem 2.3, (T, $U)$ has a faithful presentation

$$
(T,+)=\operatorname{Gp}\langle U ; \mathscr{R}\rangle .
$$

Let $F$ be the free group on $S, G$ the free group on $U$ and assume that $U=S \cup\{1\}$. Then there is a natural embedding of $F$ in $G$. Indeed we assume that $F \subseteq G$. As in Meldrum (2), Theorem 2.1, we can define $(F, S)$ and $(G, U)$ as free d.g. near-rings, using multiplication in $S$ and $U$ respectively, to define multiplication in $F$ and $G$. The natural embedding of $F$ in $G$ is a d.g. near-ring embedding. From the definition of presentations, the kernel of the d.g. homomorphism $\theta:(F, S) \rightarrow(R, S)$, extending the identity map on $S$, is $H$, the normal closure of $\mathscr{R}$ in $F$. The kernel of the d.g. homomorphism $\phi:(G, U) \rightarrow(T, U)$, extending the identity map on $U$, is $K$, the normal closure of $\mathscr{R}$ in $G$. Also $H$ is an ideal of $F$ and $K$ is an ideal of $G$.

If we now remove the assumption that $(R, S)$ is faithful, the whole of the previous work goes through with $T=\mathrm{Gp}\langle 1\rangle *(R,+)$. Then $T=\mathrm{Gp}\langle U\rangle$. The only difficulty is that we do not know whether the multiplication in $U$ can be extended to make $(T, U)$ a d.g. near-ring. This can be done if and only if $K$ is an ideal. Also $(R, S)$ is faithful if and only if $(T, U)$ is a d.g. near-ring. Hence the question as to whether we have a faithful presentation for $(R, S)$ is equivalent to the question as to whether $K$ is an ideal. As the ideal generated by a set of elements is important in this context we quote the following result from Meldrum (2), Lemma 3.2.

Lemma 2.4. Let $(R, S)$ be a d.g. near-ring, and let $X=\left\{x_{\lambda} ; \lambda \in \Lambda\right\}$ be a set of elements in $R$. Then the ideal I of $(R, S)$ generated by $X$ is a normal subgroup of $(R,+)$ 
generated by

$$
R X S=\left\{r x_{\lambda} s, r x_{\lambda}, x_{\lambda} s, x_{\lambda} ; \lambda \in \Lambda, r \in R, s \in S\right\}
$$

We now consider $(G, U)$, the free d.g. near-ring on $U$, and we wish to establish when $K$, the normal closure of $\mathscr{R}$ in $G$, is an ideal. If $K$ is an ideal, then it will be the ideal of $(G, U)$ generated by $\mathscr{R}$. By Lemma 2.4 , the ideal $I$ of $(G, U)$ generated by $\mathscr{R}$ is the normal closure of

$$
G \mathscr{R} U=\left\{g r_{\lambda} u, g r_{\lambda}, r_{\lambda} u, r_{\lambda} ; \lambda \in \Lambda, g \in G, u \in U\right\} .
$$

We do know that $H$, the normal closure of $\mathscr{R}$ in $F \subseteq G$, is an ideal of $(F, S)$. So $r_{\lambda}$ and $r_{\lambda} s$ lie in $H$ for all $\lambda \in \Lambda, s \in S$. Since $U=S \cup\{1\}$, we deduce that $r_{\lambda} u$ and $r_{\lambda}$ lie in $H \subseteq K$ for all $\lambda \in \Lambda, u \in U$. So we only need to consider elements of $G$ of the type $g r_{\lambda} u$, since $1 \in U$ and so $g r_{\lambda}=g r_{\lambda} 1$ is of this type. Then

Let $g=w(u) \in G$ be an expression for $g$ as a word in $U$. Let $r_{\lambda}=r_{\lambda}\left(s_{1}, \ldots, s_{t}\right)$.

$$
\begin{aligned}
w(\mathrm{u}) r_{\lambda} u & =w(\mathbf{u}) r_{\lambda}\left(s_{1}, \ldots, s_{t}\right) u \\
& =w(\mathbf{u}) r_{\lambda}\left(s_{1} u, \ldots, s_{t} u\right) \text { by Lemma } 2.2
\end{aligned}
$$

If $u=1$, then $r_{\lambda}\left(s_{1} u, \ldots, s_{t} u\right)=r_{\lambda}\left(s_{1}, \ldots, s_{t}\right)$. If $u \neq 1$, then $u \in S$ and so $r_{\lambda}\left(s_{1} u, \ldots, s_{t} u\right) \in H$. So we may omit $u$ in the expression above without any loss of generality. So we consider

$$
w(\mathbf{u}) r_{\lambda}\left(s_{1}, \ldots, s_{\imath}\right)=r_{\lambda}\left(w\left(\mathbf{u} s_{1}\right), \ldots, w\left(\mathbf{u} s_{t}\right)\right)
$$

This is a word in $s_{1}, \ldots, s_{t}$, since $u$ consists of elements in $U=S \cup\{1\}$. So $w(u) r_{\lambda} \in K$ if and only if $w(u) r_{\lambda} \in K \cap F=H$. The fact that $K \cap F=H$ is easy to prove. For the homomorphism $\theta$ from $G$ to $T$, extending the identity map on $U$, has kernel $K$. When restricted to $F \subseteq G, \theta$ maps $F$ onto $R \subseteq T$, with kernel $H$. Hence $K \cap F=H$. Putting all this together we get the following result.

Theorem 2.5. Let $(R,+)=\mathrm{Gp}\langle S ; \mathscr{R}\rangle$ be a presentation of the d.g. near-ring $(R, S)$, where $\mathscr{R}=\left\{r_{\lambda} ; \lambda \in \Lambda\right\}$. Then $(R, S)$ is faithful if and only if

$$
r_{\lambda}\left(w\left(u s_{1}\right), \ldots, w\left(\mathbf{u} s_{t}\right)\right) \in H
$$

for all $\lambda \in \Lambda, w(u) \in \operatorname{Fr}(S \cup\{1\})-\operatorname{Fr}(S)$, where $H$ is the normal closure in $\operatorname{Fr}(S)$ of $\mathscr{R}$, $\operatorname{Fr}(X)$ is the free group on $X$ and $r_{\lambda}=r_{\lambda}\left(s_{1}, \ldots, s_{1}\right)$.

We now apply this result to obtain a presentation of the lower faithful d.g. near-ring for a d.g. near-ring $(R, S)$.

Let $(R, S)$ be a d.g. near-ring with a presentation $(R,+)=G p\langle S ; \mathscr{R}\rangle$. Let $U=$ $S \cup\{1\}$ be the semigroup $S$ with an identity adjoined, and let $\operatorname{Fr}(U), \operatorname{Fr}(S)$ be the free groups on $U, S$ respectively. Let $H$ be defined as before, namely the normal closure of $\mathscr{R}$ in $\operatorname{Fr}(S)$. Define $H_{n}$, for $n \geqslant 1$, inductively by:

$$
H_{1}=H \text {, }
$$

$H_{n+1}$ is the normal closure in $\operatorname{Fr}(S)$ of the set $\mathscr{R}_{n+1}=\left\{h_{n}\left(w\left(u s_{1}\right), \ldots, w\left(u s_{t}\right)\right)\right.$; all $h_{n} \in H_{n}, h_{n}=h_{n}\left(s_{1}, \ldots, s_{\imath}\right)$, all $w(u)$ in $\left.\operatorname{Fr}(U)\right\}$. 
Obviously $H_{n} \subseteq H_{n+1}$ for all $n \geqslant 1$. So we can define $\bar{H}=\cup_{n=1}^{x} H_{n}$, and it is a normal subgroup of $\operatorname{Fr}(S)$. What is more, if $h \in \bar{H}$, then $h \in H_{n}$ for some $n$ and so $w(u) h \in \mathscr{R}_{n+1}$ by Lemma 2.2. So in $\operatorname{Fr}(U)$, we have $\operatorname{Fr}(U) \bar{H} \subseteq \bar{H}$. We now show that $\bar{H} S \subseteq \bar{H}$ by induction. Certainly $H S \subseteq S$ by definition of $H$, since $(R, S)$ is a d.g. near-ring. Assume that $H_{n} S \subseteq H_{n}$. Let $h_{n}\left(w\left(u s_{1}\right), \ldots, w\left(u s_{t}\right)\right) \in \mathscr{R}_{n+1}$, and let $s \in S$. Then

$$
\begin{aligned}
h_{n}\left(w\left(\mathrm{u} s_{1}\right), \ldots, w\left(\mathrm{u} s_{t}\right)\right) s & =h_{n}\left(w\left(\mathrm{u} s_{1} s\right), \ldots, w\left(\mathrm{u} s_{t} s\right)\right) \text { by Lemma } 2.2 \text { applied twice } \\
& =w(\mathrm{u}) h_{n}\left(s_{1} s, \ldots, s_{t} s\right) \text { by Lemma } 2.2 .
\end{aligned}
$$

But $h_{n}\left(s_{1} s, \ldots, s_{t} s\right)=h_{n}\left(s_{1}, \ldots, s_{t}\right) s$ lies in $H_{n}$ since $h_{n} \in H_{n}$ and $H_{n} S \subseteq H_{n}$ by the induction hypothesis. Hence

$$
w(\mathbf{u}) h_{n}\left(s_{1} s, \ldots, s_{t} s\right)=h_{n}\left(w\left(\mathbf{u} s_{1}\right), \ldots, w\left(\mathbf{u} s_{t}\right)\right)
$$

lies in $\mathscr{R}_{n+1}$. But

$$
(-x+y+x) s=-x s+y s+x s
$$

shows that since $\mathscr{R}_{n+1} S \subseteq \mathscr{R}_{n+1}$, so $H_{n+1}$, the normal closure of $\mathscr{R}_{n+1}$ in $\operatorname{Fr}(S)$, satisfies $H_{n+1} S \subseteq H_{n+1}$. Hence $\bar{H} S \subseteq \bar{H}$ by induction. We now have almost proved the following result.

Theorem 2.6. Let $(R, S)$ be a d.g. near-ring with a presentation $(R,+)=$ $\mathrm{Gp}\langle S ; \mathscr{R}\rangle$. Define $\bar{H}$ as above. Then $\bar{H}$ is an ideal in $(\operatorname{Fr}(S), S)$ and $(\operatorname{Fr}(S), S) / \bar{H}$ is the lower faithful d.g. near-ring for $(R, S)$.

Proof. By what we have done above, $\bar{H}$ is a normal subgroup of $\operatorname{Fr}(S)$, invariant under left multiplication by $\operatorname{Fr}(U)$ and right multiplication by $S$. Hence $\bar{H} U \subseteq \bar{H}$, since $U=S \cup\{1\}$. So $\bar{H}$ is an ideal of $(\operatorname{Fr}(S), S)$ and satisfies the conditions of Theorem 2.5. Hence $(\operatorname{Fr}(S), S) / \bar{H}$ is a faithful d.g. near-ring. Now suppose $(\operatorname{Fr}(S), S) / L$ is a faithful d.g. near-ring with $L \supseteq H$. Then $L \supseteq H_{1}$. Suppose $L \supseteq H_{n}$. Then $h_{n} \in L$ for all $h_{n} \in H_{n}$, and as $(\operatorname{Fr}(S), S) / L$ is faithful, $w(\mathbf{u}) h_{n} \in L$ for all $w(u) \in \operatorname{Fr}(U)$ by Theorem 2.5. But $w(\mathrm{u}) h_{n}=h_{n}\left(w\left(\mathrm{u} s_{1}\right), \ldots, w\left(\mathrm{u} s_{t}\right)\right)$, where $h_{n}=h_{n}\left(s_{1}, \ldots, s_{t}\right)$ by Lemma 2.2. Hence $\mathscr{R}_{n+1} \subseteq L$ and so $H_{n+1} \subseteq L$ as $L$ is a normal subgroup of $\operatorname{Fr}(S)$. By induction, $H_{n} \subseteq L$ for all $n \geqslant 1$ and so $\bar{H} \subseteq L$. Thus $(\operatorname{Fr}(S), S) / L$ is a homomorphic image of $(\operatorname{Fr}(S), S) / \bar{H}$. This finishes the proof of the theorem.

Although Theorems 2.5 and 2.6 are very unlikely to yield general results, they can be used to good purpose in many special cases as we will now see.

\section{Applications}

The first result we will prove is the general version of a result we have used already in two special cases, Meldrum (2), Theorem 3.3 and Meldrum (3), Theorem 2.7, and which we will use again later.

Lemma 3.1. Let $(R, S)$ be a d.g. near-ring. Suppose $S$ contains three elements $x, y, z$ such that $x+y-z=0$ in $R$. If $(R, S)$ is faithful, then $-x-y+x+y=0$.

Proof. We apply Theorem 2.5 with $w(u)=1+1$. Then $x+x+y+y-z-z=0$ in 
$R$. But $x+y-z+x+y-z=0$. Comparing these two expressions, we obtain

$$
x+y+y-z=y-z+x+y \text {. }
$$

As $y-z=-x$, this reduces to

$$
x+y-x=-x+x+y=y,
$$

which gives us our result.

We now consider a special class of d.g. near-rings for which Theorems 2.5 and 2.6 take a simpler form, and which are useful as a source of examples. We call a near-ring a zero near-ring if every product is zero. In this case all normal subgroups are ideals.

Definition 3.2. Let $G=\mathrm{Gp}\langle X ; \mathscr{R}\rangle$ be a presentation for the group $G$. The presentation is said to be integrally closed if, whenever $r=r\left(x_{1}, \ldots, x_{s}\right) \in \mathscr{R}$, then $r\left(n x_{1}, \ldots, n x_{s}\right)$ is also a relation in $G$ for all integers $n$.

This definition enables us to restate Theorem 2.5 for zero near-rings in a simple form.

Lemma 3.3. Let $(R, S)$ be a zero d.g. near-ring. Then $(R, S)$ is faithful if and only if any presentation of $R$ with generators $S$ is integrally closed.

Proof. The proof consists of showing that in the statement of Theorem 2.5, we can replace $w(u)$ by $n 1$ for all integers $n$ : Consider $w(u s)=w\left(u_{1} s, \ldots, u_{t} s\right)$ for $u_{1}, \ldots, u_{t}$ in $U$, say, where without loss of generality $u_{1}=1$ while $u_{2}, \ldots, u_{t}$ are in $S$. Then $u_{2} s=\cdots=u_{t} s=0$ and so

$$
\begin{aligned}
w(\mathbf{u} s) & =w(s, 0, \ldots, 0) \\
& =w\left(u_{1}, 0, \ldots, 0\right) s .
\end{aligned}
$$

Hence, $w(u s)=n_{w}(s)$ for some integer $n_{w}$ depending on $w(u)$. This corresponds to replacing $w(u)$ by $n_{w} 1$, where $n_{w}$ is the sum of the coefficients of 1 in the expression for $w(u)$. This is sufficient to prove the result.

This lemma also shows that in the case of an arbitrary zero d.g. near-ring, the process described just before Theorem 2.6 to obtain the lower faithful near-ring for $(R, S)$ need only consider words $w(\mathbf{u})$ of the form $n 1$, for all integers $n$.

We now consider a d.g. near-ring on a dihedral group $D_{2 n}=$ $\mathrm{Gp}\langle x, y ; 2 x=n y=x+y+x+y=0\rangle$ of order $2 n$, and we assume that the distributive generating set $S$ contains $x$ and $y$. We can say a good deal about the lower faithful d.g. near-ring for $\left(D_{2 n}, S\right)$ without any further knowledge of the structure of $S$.

Theorem 3.4. Let $(R, S)$ be a d.g. near-ring, let $x, y$ lie in $S$ and suppose that part of a presentation for $R$ is

$$
(R,+)=\mathrm{Gp}\langle x, y ; 2 x=n y=x+y+x+y=0\rangle .
$$

(i) If $n$ is odd, then the lower faithful near-ring for $(R, S)$ is $G F(2)$, the Galois field of order 2.

(ii) If $n$ is even, and $n / 2$ is odd, then the lower faithful near-ring is $D_{4}$, the ring of order 4 which is a homomorphic image of $(R, S)$. 
(iii) If 4 divides $n$, then the lower faithful near-ring for $(R, S)$ is either $D_{4}$, the ring of order 4 , or $D_{8}$ the d.g. near-ring of order 8 , in each case a homomorphic image of $(R, S)$. Both cases are possible.

Proof. Apply Theorem 2.6. In this case we choose $w(u)=1+1$, and we take $x+y+x+y$ as the relation. Then

$$
x+x+y+y+x+x+y+y=4 y=0
$$

is a relation in the lower faithful near-ring for $(R, S)$, since $x+x=0$. We also have $n y=0$.

(i) If $n$ is odd, then this forces $y=0$, and we get the result since all rings are faithful.

(ii) If $n$ is even, and $n / 2=m$, say, is odd, then this forces $2 y=0$, and again the result follows, since $D_{4}$ is abelian, and so is a ring (Pilz (4), Proposition 6.6).

(iii) If 4 divides $n$, then $D_{4}$ and $D_{8}$ are both homomorphic images of $D_{2 n}=(R,+)$. As $4 y=0$ in the lower faithful near-ring for $(R, S)$, these are the only possibilities.

We now give two examples to show that both cases in (iii) are possible. In each case the example will be a zero near-ring. In the first case we consider $\left(D_{2 n}, S\right)$ with 4 dividing $n$, and with $z=x+y$ in $S$. Then by Lemma 3.1 it follows that the images of $x$ and $y$ must commute in the lower faithful near-ring for $\left(D_{2 n}, S\right)$. This does not happen in $D_{8}$, so the lower faithful near-ring for $\left(D_{2 n}, S\right)$ is $D_{4}$ in this case.

In the second example we restrict $S$ to be only $\{x, y\}$. The presentation is then

$$
D_{8}=\mathrm{Gp}\langle x, y ; 2 x=4 y=x+y+x+y=0\rangle \text {. }
$$

In checking whether this is a faithful presentation we only need to consider $w(u)$ of the form $n 1, n$ an integer, as we showed in Lemma 3.3. Obviously we get nothing new from $2 n x$ and $2 n y$, in the process described in Theorem 2.6. When we consider $x+y+x+y$ we obtain $n x+n y+n x+n y$. If $n$ is even, then $n x=0$, and this word reduces to $2 n y$, with 4 dividing $2 n$. If $n$ is odd, then this word reduces to $x+n y+x+$ $n y$ which is true in $D_{8}$ already. Hence the presentation of $D_{8}$ is integrally closed and $D_{8}$ is the lower faithful near-ring for $\left(D_{2 m}, S\right)$, if 4 divides $m$.

The final application concerns d.g. near-rings $(R, S)$ where $R$ is a finite simple non-abelian group. By Pilz (4), Theorem 9.102, $R$ is either a zero near-ring or has an identity. By Pilz (4), Corollary $9.115(b)$ the second case cannot happen. So $(R, S)$ is a zero near-ring.

Theorem 3.5. Let $(R, S)$ be a d.g. near-ring where $(R,+)$ is a finite simple non-abelian group. Then $R$ is a zero d.g. near-ring. If the presentation for $R$ is integrally closed, then $(R, S)$ is faithful. If the presentation for $R$ is not integrally closed, then the lower faithful near-ring for $(R, S)$ is $\{0\}$.

Proof. We have shown above that $(R, S)$ is a zero d.g. near-ring. Then, by Lemma 3.3, $(R, S)$ is faithful if and only if the presentation for $R$ is integrally closed. If $(R, S)$ is not faithful, then the lower faithful near-ring for $(R, S)$ is a proper homomorphic image of $(R, S)$. As $(R,+)$ is simple, this can only be $\{0\}$. This finishes the proof.

Each case can occur. We can use Lemma 3.1 to get the second case, as was done 
in Meldrum (3), Theorem 2.7. On the other hand we can take $S$ to consist entirely of elements of order 2 . If $r\left(s_{1}, \ldots, s_{t}\right)$ is a relation, then $r\left(n s_{1}, \ldots, n s_{t}\right)$ is either $r(0, \ldots, 0)=0$ if $n$ is even, or is $r\left(s_{1}, \ldots, s_{t}\right)$ if $n$ is odd, which is already a relation. So the presentation is integrally closed in this case. This may well be the only integrally closed presentation for a finite non-abelian simple group, but we do not know the answer yet.

\section{REFERENCES}

(1) S. J. MAHMOOD, Limits and colimits in categories of d.g. near-rings, Proc. Edinburgh Math. Soc. 23 (1980), 1-7.

(2) J. D. P. MELDRUM, The representation of d.g. near-rings, J. Austral. Math. Soc. 16 (1973), 467-480.

(3) J. D. P. Meldrum, On the structure of morphism near-rings, Proc. Royal Soc. Edinburgh 81A (1978), 287-298.

(4) G. PILZ, Near-rings (North Holland, Amsterdam, 1976).

UNIVERSITY OF EDINBURGH 